\title{
COVID-19, microangiopathy, hemostatic activation, and complement
}

\author{
Wen-Chao Song ${ }^{1,2}$ and Garret A. FitzGerald ${ }^{1,2,3}$ \\ 'Department of Systems Pharmacology and Translational Therapeutics, ${ }^{2}$ Institute for Translational Medicine and Therapeutics, and ${ }^{3}$ Department of Medicine, Perelman School of Medicine, University of \\ Pennsylvania, Philadelphia, Pennsylvania, USA.
}

$T_{\text {t }}$ he COVID-19 pandemic caused by the SARS-CoV-2 virus has swept the globe with devastating societal consequences and has placed tremendous stress on health care systems. Although severe respiratory disease is a dominant feature, strokes, venous thrombosis, renal failure, cardiomyopathy, and coronary and systemic vasculitis have complicated the clinical phenotype (1-5). Indeed, besides typical features of acute respiratory distress syndrome (ARDS), patients with COVID-19 exhibit a thrombotic necrotizing injury of the lung capillaries (6). This clinical pattern, together with a low blood platelet count (thrombocytopenia) and elevated plasma fibrin degradation products (D-dimers) $(7,8)$, is highly suggestive of complement activation.

\section{Complement and its dysregulation in human disease}

The complement system is part of the innate immune system and can be activated via three separate pathways: the Ab-dependent classical pathway, the mannose-binding lectin (MBL) pathway, and the alternative pathway (Figure 1 and ref. 9). Activated complement can produce direct effector functions by target opsonization with cleaved complement component 3 (C3) and C4 fragments, promotion of inflammation with $\mathrm{C} 3 \mathrm{a}$ and C5a, and direct cell lysis with the assembly of the membrane attack complex (MAC) C5b-9 (Figure 1 and ref. 9). Complement activation also primes adaptive immune responses. For example, the adjuvant effect of $\mathrm{C} 3$ activation in eliciting a robust $\mathrm{Ab}$ response is well established (9).
Our understanding of inappropriate complement activation in human disease derives mainly from genetic mutations of complement proteins - either loss-offunction mutations in regulatory proteins that protect host tissues under homeostatic conditions or gain-of-function mutations resulting in resistance to regulatory protein surveillance (10). Paroxysmal nocturnal hemoglobinuria (PNH), for example, is caused by deficiency of 2 complement regulatory proteins on affected blood cells, decay-accelerating factor (DAF, also known as CD55) and CD59, and is characterized by complement-mediated hemolysis and multiorgan thrombosis (11). The thrombotic microangiopathy (TMA) of the atypical hemolytic uremia syndrome (aHUS) leads to thrombocytopenia, hemolytic anemia, and renal failure (12). However, thrombotic events in aHUS are not confined to the kidney; $3 \%$ to $10 \%$ of patients have cardiac complications due to coronary microangiopathy, and patients often develop complications involving other organs (12). The most frequent mutations in aHUS are found in the regulatory protein factor $\mathrm{H}(\mathrm{FH})$, and such mutations lead to MAC-mediated endothelial injury and capillary thrombosis, which are the hallmarks of TMA (13). Both PNH and aHUS are caused by inadequate control of complement activation and successfully treated with eculizumab, a humanized anti-C5 mAb (9).

\section{Evidence and mechanism of complement activation in COVID-19}

It is highly plausible that complement activation plays a role in the pathogenesis of

Conflict of interest: Conflict of interest WCS owns equity in, receives consultant fees and research grants from, and is an inventor on patents for anti-complement mAbs (W02018/165062, W02018/148486, and US2020/0148754A1) and gene therapy (W02017/053732A2), licensed to Kira Pharmaceuticals and Aevitas Therapeutics. GAF is an advisor to Calico Laboratories, from which he receives salary and research support.

Copyright: ( 2020, American Society for Clinical Investigation.

Reference information: J Clin Invest. 2020;130(8):3950-3953. https://doi.org/10.1172/JCI140183.

COVID-19. Pathogen infection is a common external trigger of increased complement activation. Although complement, like other innate immune system components, helps to control initial bacterial or viral infections, there is a risk of complement becoming detrimental in later stages of the infection because of overactivation directly induced by the pathogen, or secondarily via damaged host tissues. Runaway complement activation may overwhelm host cell regulatory mechanisms, particularly in individuals with genetic predispositions to subclinical complement regulation insufficiency.

The clinical phenotypes of COVID-19 likely reflect direct effects of virus-mediated physical damage to tissue integrity as well as maladaptive host immune responses. For example, endothelial cells express high levels of angiotensin-converting enzyme 2 (ACE2), the receptor for SARS-CoV-2, and viral infection can disrupt endothelial cell function directly or evoke an inflammatory or other immune response (14). These last mechanisms are critical in the cytokine storm that complicates the most severe phenotype of COVID-19 (15). The anaphylatoxins C3a and C5a are likely major contributors to cytokine storm syndrome, both directly through their intrinsic proinflammatory activities in leukocyte activation and trafficking and indirectly by synergizing with other innate immune sensors, such as TLRs, to amplify inflammation (16). Indeed, in a Chinese cohort of patients with COVID-19, plasma C5a levels reflected the severity of the disease (17). Anecdotally, treating two of these patients with an anti-C5a mAb coincided with resolved fever and increased oxygen saturation (17).

It is likely that complement is activated in COVID-19 via multiple pathways, both by SARS-CoV-2 itself and by damaged tissues and dying cells at later stages of the disease (Figure 1). For example, natural 


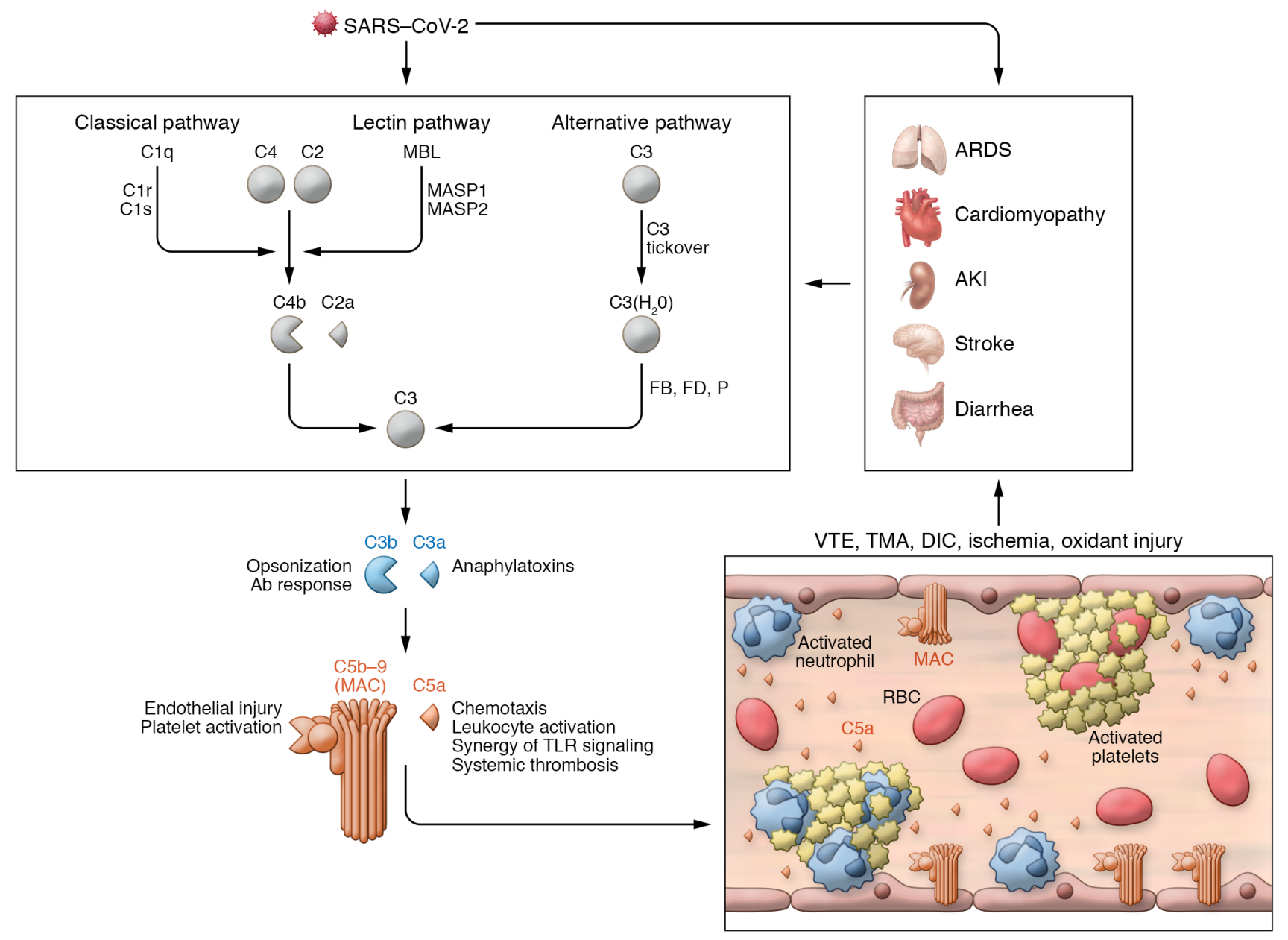

Figure 1. Potential mechanisms of complement-mediated pathology in COVID-19. SARS-CoV-2 virus may directly activate the complement pathways. Damaged host tissues could also secondarily activate complement via any of the three pathways: the classical pathway, the lectin pathway, or the alternative pathway. Complement activation generates the anaphylatoxins C3a and C5a and the MAC C5ab-9. The C5a anaphylatoxin can cause neutrophil and monocyte activation, promote the formation of NET and platelet-leukocyte aggregates, and stimulate neutrophil degranulation and the release of tissue factor to trigger the extrinsic coagulation pathway. These C5a effects may contribute to a hypercoagulative state leading to VTEs and disseminated intravascular coagulation (DIC). The MAC C5b-9 can cause direct endothelial injury and platelet activation, leading to TMA. Capillary and blood vessel occlusion by TMA and VTEs eventually results in tissue ischemia and oxidant injury, contributing to multiorgan failure in COVID-19. AKI, acute kidney injury; FB, complement factor B; FD, complement factor $\mathrm{D}$; P, properdin.

(IgM) Abs that recognize viral antigens or neoantigens exposed on damaged host tissues could trigger the classical pathway (18). The lectin pathway could also activate in response to viral components such as the nuclear protein ( $\mathrm{N}$ protein), as demonstrated in a recent study for SARS$\mathrm{CoV}$ in which the $\mathrm{N}$ protein directly interacted with and activated MBL-associated proteases 2 (MASP2) (17). Finally, dying cells in multiple ischemic organs likely shed lipid-anchored membrane complement regulatory proteins, such as DAF and CD59, and lose glycosaminoglycans for FH binding $(19,20)$, allowing complement activation by the alternative pathway to occur unimpeded.

\section{Complement-mediated coagulopathy and} microangiopathy in COVID-19

Complement activation may also contribute to hemostatic activation leading to coagulopathy and explain microvascular injury, pathological features that are widely reported in COVID-19 (4-7). In the atypical ARDS that complicates COVID-19, substantial deposits of complement C4d, MASP2, and C5b-9 have been reported in the lung and in the dermal microvasculature (6). Thus, there is evidence of systemic thrombophilia as well as microvascular injury, in parallel with elevated plasma $\mathrm{C} 5 \mathrm{a}$ and endothelial C5b-9 deposits in COVID-19.
Eculizumab effectively inhibits C5 to block venous thromboembolic events (VTEs) in PNH and TMA in aHUS (9). Until recently, the relative contributions of the C5a and C5b-9 pathways to these pathologies remained unknown. Experiments in a mouse model carrying an FH point mutation and developing both VTEs and TMA have partially elucidated this question (21). Blocking C5 in the mutant mice rescued the renal and ocular TMA as well as VTE phenotypes and prevented thrombocytopenia, hemolytic anemia, renal failure, stroke, and sudden death (22). On the other hand, when the researchers deleted the $\mathrm{C} 5 \mathrm{a}$ receptor $(\mathrm{C} 5 \mathrm{aR})$ in the same mutant FH back- 
ground, the mice avoided VTEs, but were still susceptible to TMA in the kidney and eye $(22,23)$. Conversely, blocking C5b-9 by $\mathrm{C} 6$ gene deletion rescued the mutant mice from TMA injury, but had no impact on the incidence of VTEs $(22,23)$. Thus, at least in this murine model, microvascular injury and capillary thrombosis are mediated by the MAC pathway, whereas systemic thrombophilia depends on the C5a/ C5aR pathway. The mechanism by which $\mathrm{C} 5 \mathrm{aR}$ activation induces VTEs remains unclear. However, given that neutrophils and monocytes prominently express the $\mathrm{C} 5 \mathrm{aR}$ on their cell surface, downstream contributors such as cytokine production (16), neutrophil extracellular traps (NETs) leading to platelet-leukocyte aggregates $(24,25)$, and tissue factor release to trigger the extrinsic coagulation pathway (26) could all have played a role.

\section{Considerations in anticomplement therapy for COVID-19}

The approved drugs eculizumab and RUCONEST, a C1 inhibitor (C1INH), and experimental anti-C5a and anti-C3 drugs have been administered and well tolerated under compassionate use in patients with COVID-19 (27-30). As controlled clinical trials of complement inhibitors are initiated or considered for COVID-19, it will be necessary to ask which interventions are most likely to be safe and effective at various stages of disease evolution.

C5 blockade may benefit patients who are in critical condition requiring ICU care. Here, there is evidence from mice and humans consistent with tissue damage mediated by both C5a and C5b-9 (6, 17, 22, 23). In patients with less severe disease who require hospitalization but not ICU care, an argument could also be made for prescribing medications that block $\mathrm{C} 5$, although the anti-C5a approach would carry a lower risk of Neisseria bacterial infection, which is normally controlled by MAC (9). Ideally, this question of comparative safety and efficacy would be addressed by a randomized, controlled comparison of anti-C5 versus anti-C5a mAbs, perhaps enriching patient selection by including those with elevated D-dimers and indices of complement activation. Vaccination and prophylactic use of antibiotics could mitigate the risk of Neisseria infection and consequent meningitis $(9,27)$.
However, the relative safety and comparative advantage of such strategies in patients with COVID-19 are unknown. A disadvantage of the current anti-C5 or C5a mAbs is the high dosage requirement $(9,17)$, which may create pharmacokinetic uncertainties in the setting of hemodynamic instability. In addition to these terminal complement inhibitors, drugs targeting earlier steps or a specific pathway of the complement cascade are also in clinical development (9). Such drugs may achieve more complete inhibition of complement than anti-C5 or anti-C5a, but their benefit and risk profiles also require careful consideration.

\section{Conclusions}

In summary, there are persuasive lines of evidence implicating complement activation in COVID-19. These rest on the consistency of the clinical phenotypes with both human syndromes and mouse models of complement activation. Pharmacological inhibitors of complement activation include approved drugs and others in late stages of development. Selection among these drugs and the timing of treatment will depend on controlled trials that define the relative importance of distinct complement pathways, the efficiency of drug administration, and the risk of opportunistic infection.

\section{Acknowledgments}

Research in the laboratory of WCS is supported by NIH grants AIO85596, AI117410, and AI146162. GAF is supported by NIH grants HL141912 and U54TR001623 and a Merit Award from the American Heart Association. We thank Takashi Miwa and Damodar Gullipalli for reference checking and figure preparation, and Claire Song for editorial help. GAF acknowledges the advice of Keith Peters on the potential role of complement activation in COVID-19. GAF is the McNeil Professor in Translational Medicine and Therapeutics.

Address correspondence to: Wen-Chao Song, Perelman School of Medicine, 421 Curie Boulevard, Room 1254 BRBII/III, University of Pennsylvania, Philadelphia, Pennsylvania 19104, USA. Phone: 215.573.6641; Email: Songwe@pennmedicine.upenn.edu. Or to: Garret A. FitzGerald, Institute for Translational Medicine and Therapeutics, Smilow Center for Translational Research,
West Pavilion, 10th Floor, Room 116, 3400 Civic Center Boulevard, Building 421, Philadelphia, Pennsylvania 19104-5158, USA. Phone: 215.898.1185; Email: garret@ upenn.edu.

1. Guan WJ, et al. Clinical characteristics of coronavirus disease 2019 in China. $N$ Engl J Med. 2020;382(18):1708-1720.

2. Goyal P, et al. Clinical characteristics of Covid19 in New York City [published online April 17, 2020]. N Engl J Med. https://doi.org/10.1056/ nejmc2010419.

3. Grasselli G, et al. Baseline characteristics and outcomes of 1591 patients infected with SARSCoV-2 admitted to ICUs of the Lombardy region, Italy. JAMA. 2020;323(16):1574-1581.

4. Llitjos JF, et al. High incidence of venous thromboembolic events in anticoagulated severe COVID-19 patients [published online April 22, 2020]. J Thromb Haemost. https://doi. org/10.1111/jth.14869.

5. Helms J, et al. Neurologic features in severe SARS-CoV-2 infection [published online April 15, 2020]. N Engl JMed. https://doi.org/10.1056/ nejmc2008597.

6. Magro C, et al. Complement associated microvascular injury and thrombosis in the pathogenesis of severe COVID-19 infection: a report of five cases. Transl Res. 2020;S19315244(20):30070-0.

7. Zhang L, et al. D-dimer levels on admission to predict in-hospital mortality in patients with Covid-19 [published online April 19, 2020]. J Thromb Haemost. https://doi.org/10.1111/jth.14859.

8. Yang X, et al. Thrombocytopenia and its association with mortality in patients with COVID-19 [published online April 17, 2020]. J Thromb Haemost. https://doi.org/10.1111/jth.14848.

9. Morgan BP, Harris CL. Complement, a target for therapy in inflammatory and degenerative diseases. Nat Rev Drug Discov. 2015;14(12):857-877.

10. Wong EKS, Kavanagh D. Diseases of complement dysregulation-an overview. Semin Immunopathol. 2018;40(1):49-64.

11. Brodsky RA. Paroxysmal nocturnal hemoglobinuria. Blood. 2014;124(18):2804-2811.

12. Noris M, Remuzzi G. Atypical hemolytic-uremic syndrome. $\mathrm{N} \mathrm{Engl} \mathrm{J} \mathrm{Med.}$ 2009;361(17):1676-1687.

13. Kavanagh D, Goodship T. Genetics and complement in atypical HUS. Pediatr Nephrol. 2010;25(12):2431-2442.

14. Varga Z, et al. Endothelial cell infection and endotheliitis in COVID-19. Lancet. 2020;395(10234):1417-1418.

15. Ye Q, Wang B, Mao J. The pathogenesis and treatment of the 'cytokine storm' in COVID-19. J Infect. 2020;80(6):607-613.

16. Zhang X, et al. Regulation of Toll-like receptor-mediated inflammatory response by complement in vivo. Blood. 2007;110(1):228-236

17. Gao T, et al. Highly pathogenic coronavirus $\mathrm{N}$ protein aggravates lung injury by MASP-2mediated complement over-activation [preprint]. Posted on medRxiv April 7, 2020. https:// doi.org/10.1101/2020.03.29.20041962. 
18. Zhang M, Carroll MC. Natural IgM-mediated innate autoimmunity: a new target for early intervention of ischemia-reperfusion injury. Expert Opin Biol Ther. 2007;7(10):1575-1582.

19. Jones J, Morgan BP. Apoptosis is associated with reduced expression of complement regulatory molecules, adhesion molecules and other receptors on polymorphonuclear leucocytes: functional relevance and role in inflammation. Immunology. 1995;86(4):651-660.

20. Ihrcke NS, Wrenshall LE, Lindman BJ, Platt JL. Role of heparan sulfate in immune systemblood vessel interactions. Immunol Today. 1993;14(10):500-505.

21. Ueda Y, et al. Murine systemic thrombophilia and hemolytic uremic syndrome from a factor $\mathrm{H}$ point mutation. Blood. 2017;129(9):1184-1196.

22. Ueda Y, et al. Differential contribution of C5aR and C5b-9 pathways to renal thrombic microangiopathy and macrovascular thrombosis in mice carrying an atypical hemolytic syndrome-related factor H mutation. Kidney Int. 2019;96(1):67-79.

23. Song D, et al. Complement Factor H Mutation W1206R Causes Retinal Thrombosis and Ischemic Retinopathy in Mice. Am J Pathol. 2019;189(4):826-838.

24. Cerletti C, Tamburrelli C, Izzi B, Gianfagna F, de Gaetano G. Platelet-leukocyte interactions in thrombosis. Thromb Res. 2012;129(3):263-266.

25. Zuo Y, et al. Neutrophil extracellular traps in COVID-19. JCI Insight. 2020;5(11):e138999.

26. Ritis K, et al. A novel C5a receptor-tissue factor cross-talk in neutrophils links innate immunity to coagulation pathways. JImmunol. 2006;177(7):4794-4802.

27. Pitts TC, et al. A preliminary update to the
Soliris To Stop Immune Mediated Death in Covid-19 (SOLID-C19) compassionate use study. https://hudsonmedical.com/articles/ soliris-stop-death-covid-19. Updated April 9, 2020. Accessed May 30, 2020.

28. Pharming. Pharming reports encouraging results from use of RUCONEST ${ }^{\circledR}$ in COVID-19 patients. https://www.pharming.com/news/pharming-reports-encouraging-results-use-ruconestr-covid-19-patients. Updated April 21, 2020. Accessed May 20, 2020.

29. Diurno F, et al. Eculizumab treatment in patients with COVID-19: preliminary results from real life ASL Napoli 2 Nord experience. Eur Rev Med Pharmacol Sci. 2020;24(7):4040-4047.

30. Mastaglio S, et al. The first case of COVID-19 treated with the complement C3 inhibitor AMY101. Clin Immunol. 2020;215:108450. 